\title{
The Revision of the Alleged Spherical Atom Model of Helium
}

\author{
Thomas Allmendinger \\ Independent Scholar, Zurich, Switzerland \\ Email: inventor@sunrise.ch
}

How to cite this paper: Allmendinger, T. (2019) The Revision of the Alleged Spherical Atom Model of Helium. Journal of Applied Mathematics and Physics, 7, 1212-1219. https://doi.org/10.4236/jamp.2019.75081

Received: March 11, 2019

Accepted: May 27, 2019

Published: May 30, 2019

Copyright $\odot 2019$ by author(s) and Scientific Research Publishing Inc. This work is licensed under the Creative Commons Attribution International License (CC BY 4.0).

http://creativecommons.org/licenses/by/4.0/

\section{c) (i) Open Access}

\begin{abstract}
The purpose of this contribution was to evaluate a recently published atom model for Helium, characterized by a double rotation of the electrons which exhibit perpendicular rotation axes. Thereby, each rotation is induced by the spin of one electron [1]. Hereto, a tangible mechanical model was used which facilitated to derive the mathematical formulae as the basics for two-dimensional projections, and-not least-for a digital animation yielding freeze images from different perspectives. The resulting shape of the electron shell turned out to be not spherical. In particular, the total velocity of the electrons is variable since the relative running direction may change-in contrast to the initial assumption-, even leading to an intermittent standstill, and implying a variable kinetic energy. Thus it can be concluded that this model describes a rotating rotor but not the Helium atom, and that it must be abandoned.
\end{abstract}

\section{Keywords}

Electron-Trajectories, 2D-Projections, Tangible Mechanic Atom-Model, 3D-Animation, Rotating Rotor

\section{Introduction}

According to the conventional theory of quantum mechanics, the electron trajectories in atoms and in molecules are assumed as indeterminable and solely describable by probabilities of presence, implying Heisenberg's uncertainty principle. In the case of Helium-the element with the most simple singular atoms-, the symmetry of the double-occupied 1s orbital is assumed as strictly spherical, exhibiting no asymmetry or anisotropy. The charge cloud model proposed by Kimball [2] is clearer but not exactly computable.

In contrast to this atom- and molecule-model where probabilities of presence are assumed, the author's alternative approach is based on the assumption of 
well-defined electron trajectories. It proceeds from Bohr's planar hydrogen model, published in 1913 [3], wherein a fixed orbital angular momentum is postulated for the ground state, determined by Planck's constant $h / 2 \pi$. But in addition, the electron spin-discovered in 1925/26 by Uhlenbeck and Goudsmith [4] [5] —is adduced to explain the existence of this ground state, inducing an invariable orbital angular momentum of the electron (=radius $\times$ velocity $\times$ mass), due to a resonance effect, and leading to the-empirically detectable-spin-orbit coupling.

As the author could point out in [6], it is possible to precisely compute the bond distance in the $\mathrm{H}_{2}$-molecule known from X-ray-spectroscopic measurements, using normal physical laws and the condition of the constant orbital momentum for each electron. In the next step, a 3D atom model for the noble gases Helium and Neon was conceived [7] in light of the fact that a Helium model is more speculative than the $\mathrm{H}_{2}$-model since no precise empiric data exist for its verification. Therein, the two electron orbits of the first shell were arranged as double cones, cf. Figure 1. Surpassing this, a further approach was made which promised to be more spherical [1], cf. Figure 2. However, that electron shell turned out to be not easily describable. As a consequence, a more profound evaluation of this approach seemed to be indicated, using a tangible

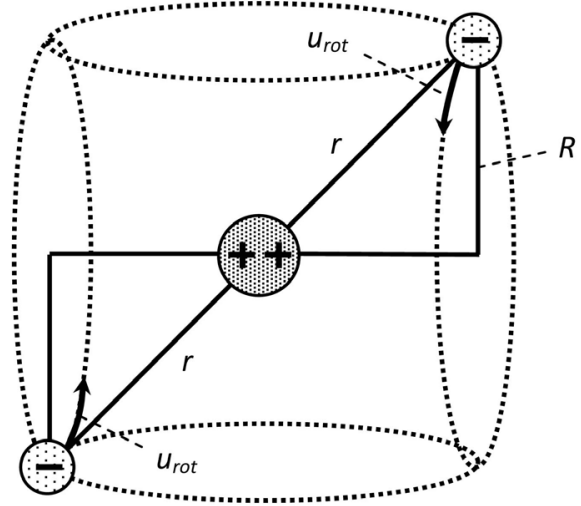

Figure 1. Schematic figure of the double-cone model of Helium, according to [7].

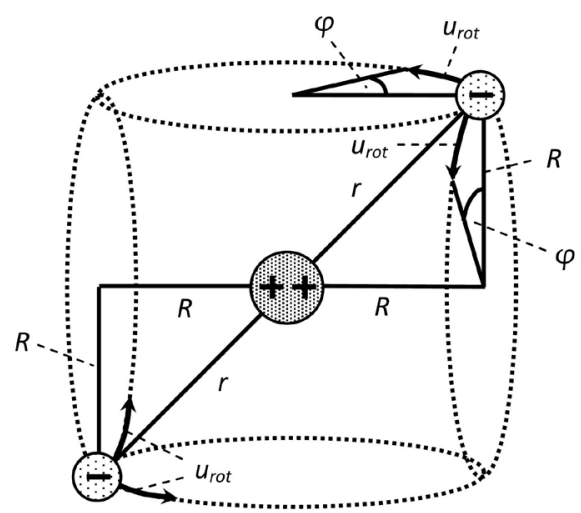

Figure 2. Schematic figure of the rotating rotator atom model for Helium with the rotating radius $R$, the rotating angle $\varphi$, and the rotating velocity $u_{\text {roo }}$ according to [1]. 
mechanical model which facilitated to derive the mathematical formulae as the basics for two-dimensional projections, and-not least-for a digital animation yielding freeze frames from different perspectives.

\section{The Tangible Mechanic Model of the Helium Atom}

As outlined in [1], the here proposed three-dimensional atomic model of Helium is characterized by a double rotation of the electrons which exhibit perpendicular rotation axes. Thereby, each rotation is induced by the spin of one electron. Thus the trajectory of each electron represents the superposition of two separate orbits, while each electron is always positioned opposite to the other one (Figure 2). Both electron velocities were assumed as counter-current and identically equal, due to the mutual coupling, implicating a permanent total velocity according to $\left(u_{\mathrm{tot}}\right)^{2}=2\left(u_{\mathrm{rot}}\right)^{2}$.

In order to visualise the resulting electron trajectories, it was advantageous to construct a tangible mechanic model which can be manually stirred. Thereto, the kit of Stokys was used. The here applied model is shown from two different perspectives and at two different rotation angles in Figure 3 and Figure 4. The definition of the axis-directions is given in Figure 5.

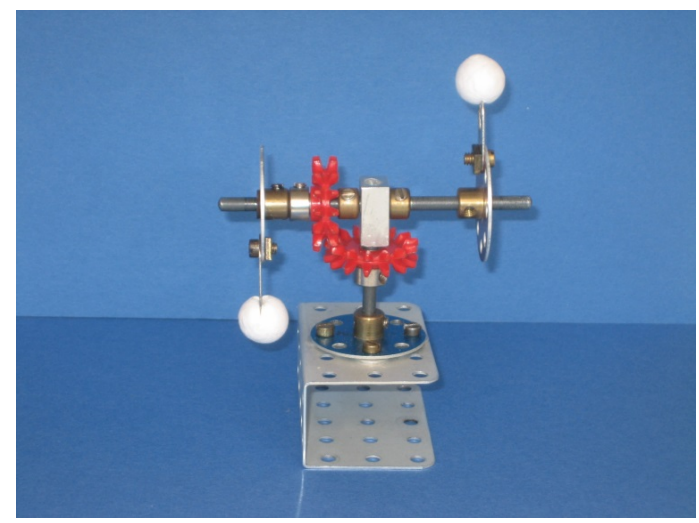

Figure 3. Stokys-model for the electron positions (white spheres) from the $\mathrm{x}$-axis perspective according to Figure 5.

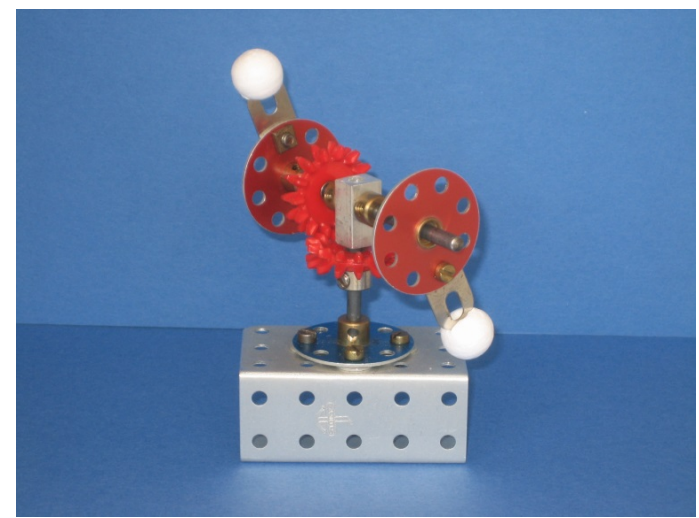

Figure 4. Stokys-model for the electron positions (white spheres) from the $\mathrm{z}$-axis perspective according to Figure 5. 


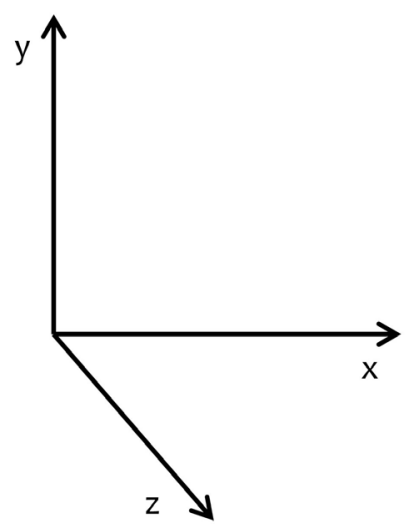

Figure 5. Definition of the axis-directions, consistent with the view shown in Figure 4.

\section{Derivation of the Angle Dependent Position of the Electrons}

In order to 2D-describe the electron trajectories in a Cartesian coordinate system (defined in Figure 5) with the rotation angle $\varphi$ as a variable, their $x^{-}, y^{-}$and $z$-projections on the $x / y$-plane may be used as parameters. Applying descriptive geometry and using the tangible mechanic model as an aid, for one electron the analysis yields

$$
x=R \cdot \sin \varphi(1+\cos \varphi), \quad y=-R \cdot \cos \varphi, \quad z=R\left(\cos \varphi-(\sin \varphi)^{2}\right)
$$

For the other electron, the formulas are identical equal except the sign. The respective diagrams for the two electrons are shown in the Figure 6 and the Figure 7.

Note: These formulas are analogous to those given in [1], but they differ with respect to the signs and the mappings due to another definition of the coordinate system.

\section{Trajectory-Projections on the Different Planes of the Coordinate System}

The availability of the $x / y / z$-parameters as a function of the rotation angle enables to easily determine the trajectory projections on the three possible planes, namely the $x / y$-, the $x / z$ - and the $y / z$-plane. They are displayed in Figures 8-10. The respective shapes are exceptional and do not resemble to any known geometric figures. Obviously, they exhibit sharpened points which indicate standstills. Thus the motion is not continuous and constant.

\section{3D-Animations and Their Freeze Images}

In order to visualize the three dimensional shape of the electron trajectories, 3D-animations were made using a VPython computer program. The resulting images strongly depend on the-virtual-camera positions, implying different perspectives. From the numerous possibilities, the four examples shown in Figures 11-14 as freeze images were chosen. 


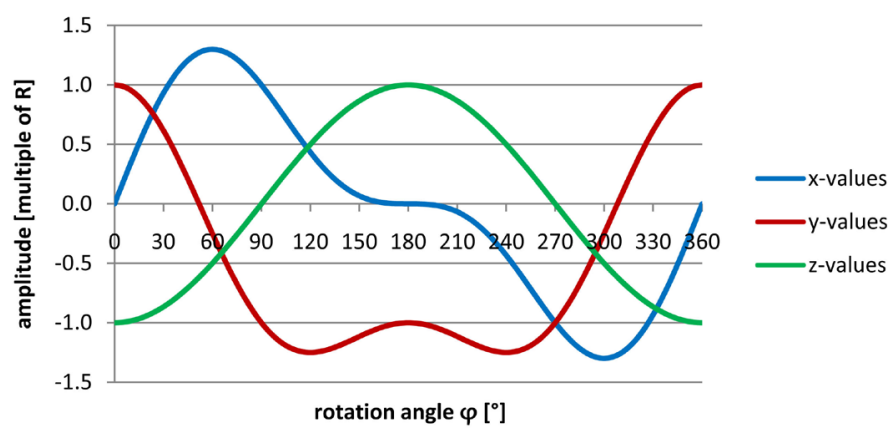

Figure 6. The axis-projections for one electron as a function of the rotation angle.

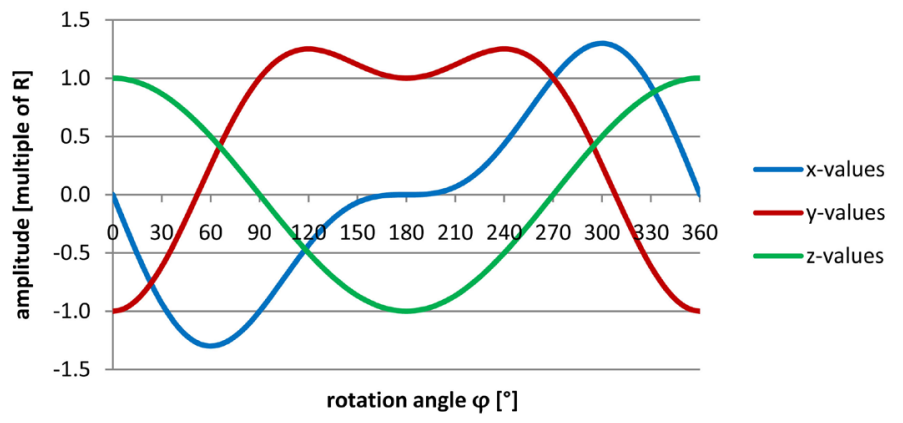

Figure 7. The axis-projections for the other electron as a function of the rotation angle.

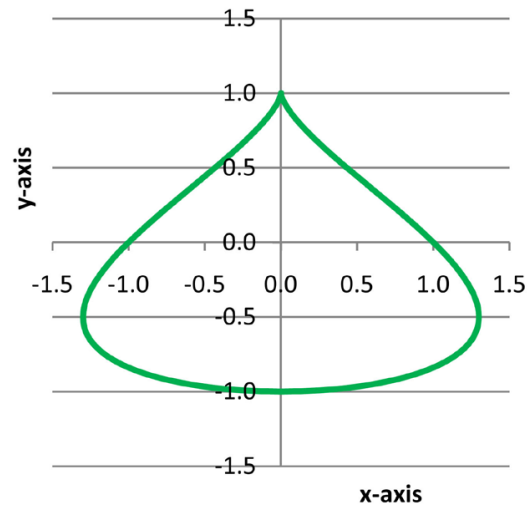

Figure 8. Trajectory-projection on the $x / y$-plain.

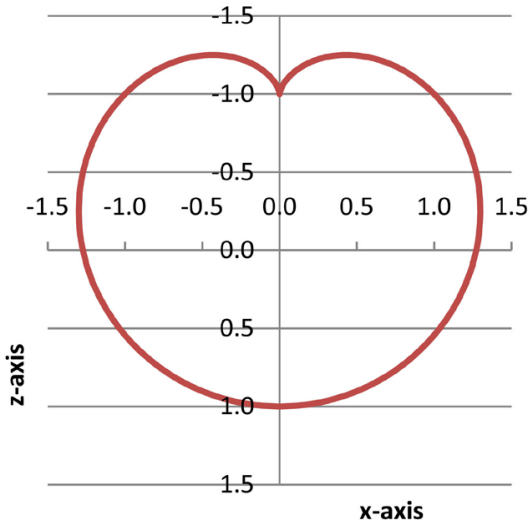

Figure 9. Trajectory-projection on the $x / z$-plain. 


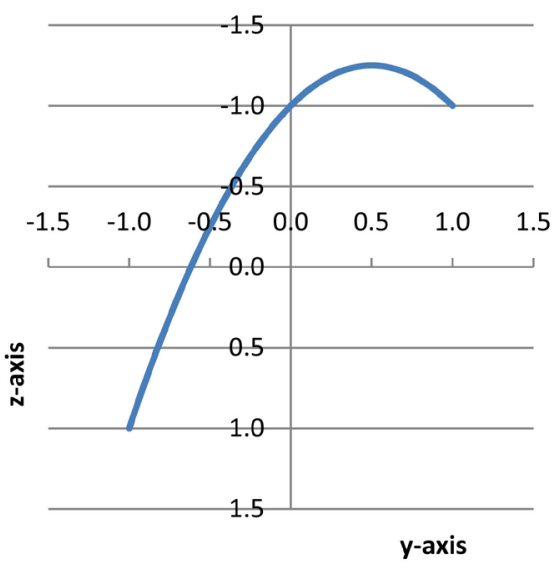

Figure 10. Trajectory-projection on the $y / z$-plain.

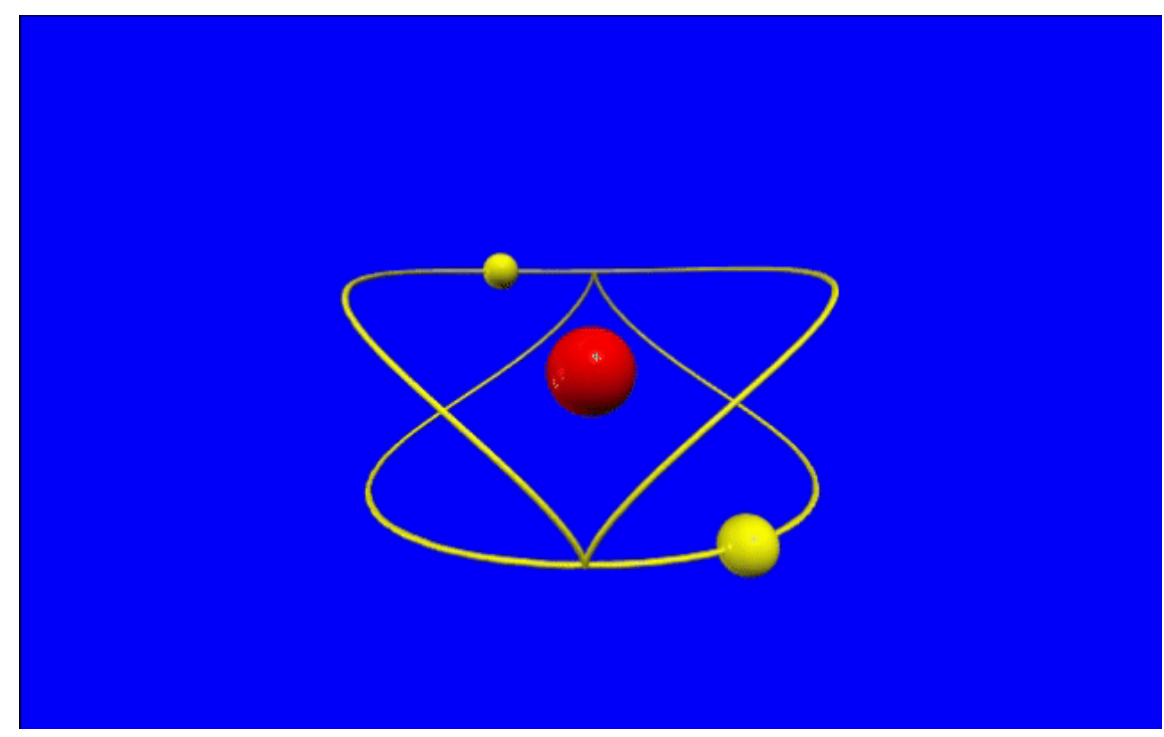

Figure 11. Camera-position: $x=2.2 / y=-2.0 / z=10.9$.

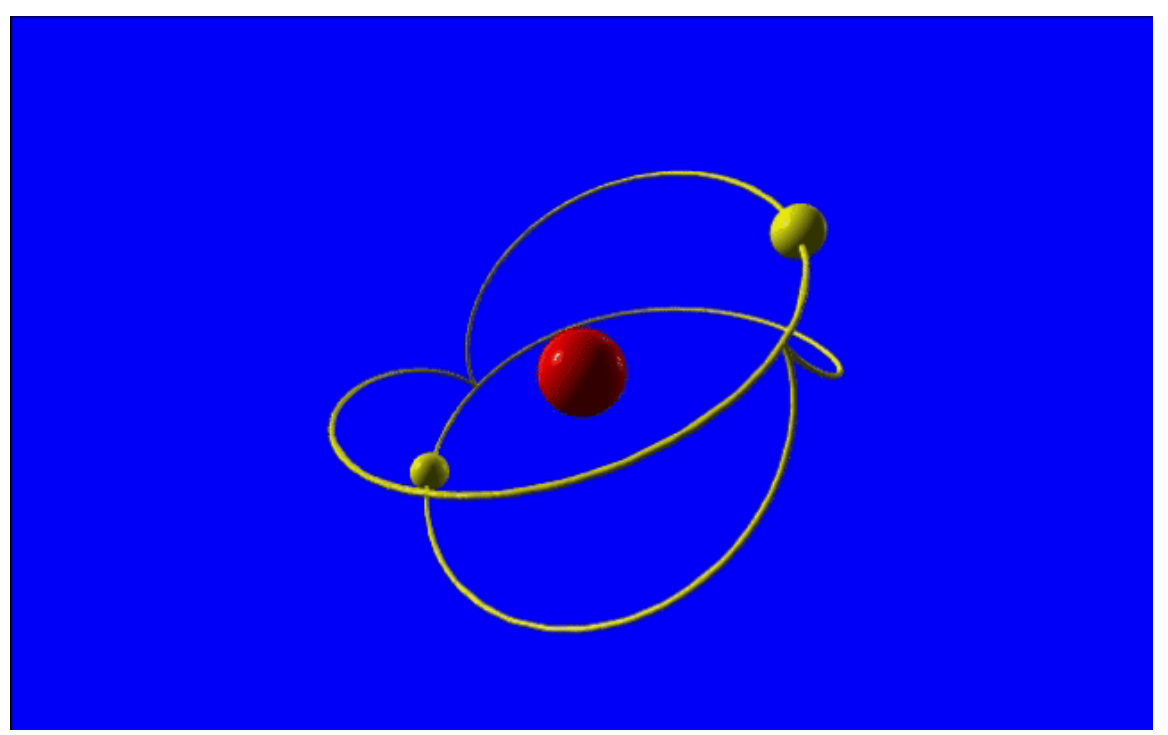

Figure 12. Camera-position: $x=5.0 / y=9.0 / z=-4.0$. 


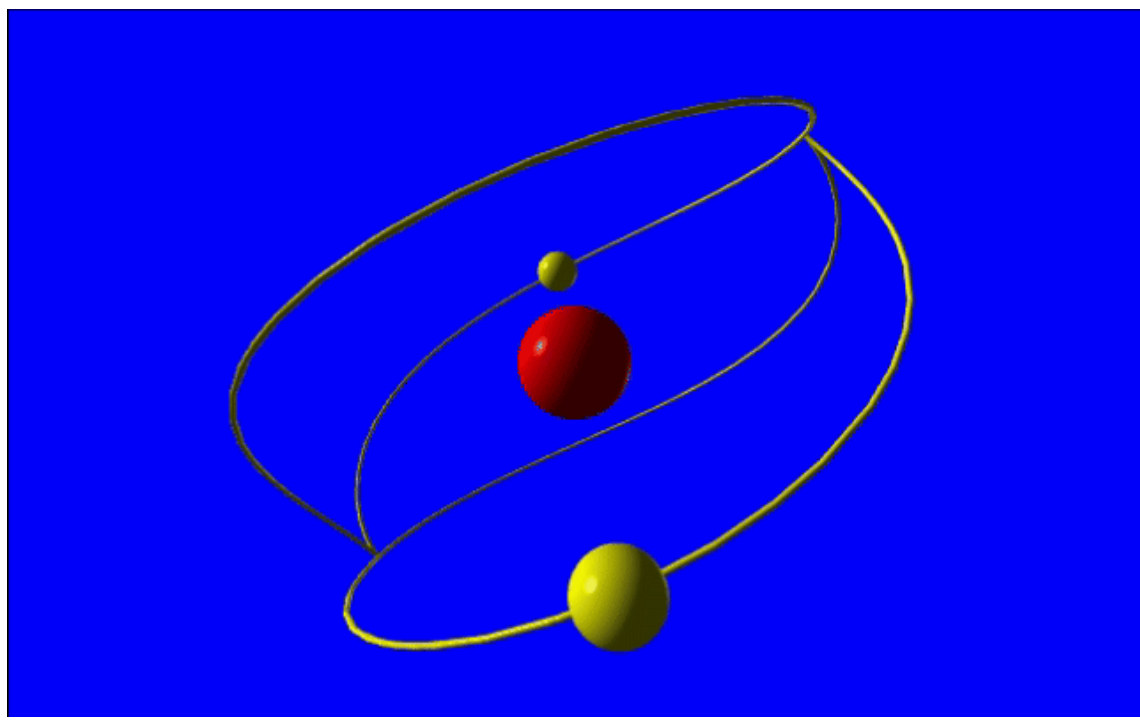

Figure 13. Camera position: $x=11.0 / y=0.0 / z=0.0$.

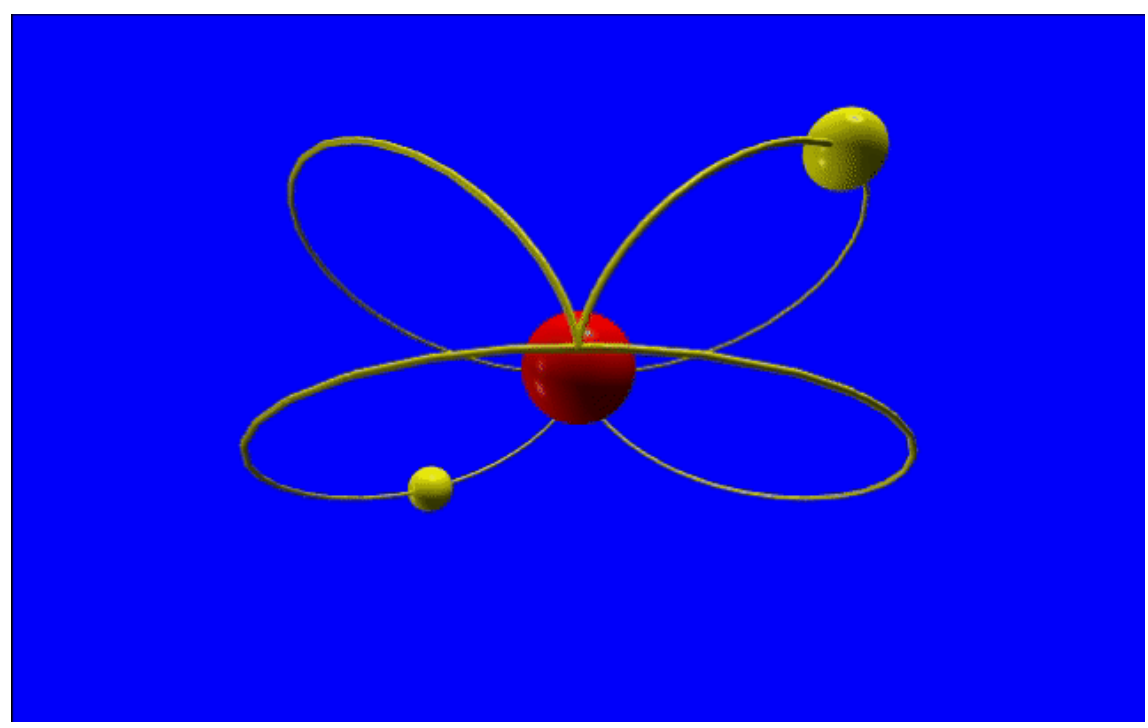

Figure 14. Camera-position: $x=0.1 / y=-7.8 / z=7.9$.

\section{Summary and Conclusion}

The here presented figures yield a deepened and clearer comprehension of the recently published atom model of Helium [1]. For tracing the electron motions, a tangible mechanic model was used, enabling the derivation of formulas for the Cartesian coordinate-intercepts by applying descriptive geometry. The formulas provide $2 \mathrm{D}$-depictions of the electron trajectories and, in particular, 3D-ones as freeze images of animations. The resulting shape of the electron shell is three-dimensional but not spherical. In particular, the total velocity of the electrons is variable since the relative running direction may change-in contrast to the initial assumption-, even leading to an intermittent standstill, and implying a variable kinetic energy. Thus it can be concluded that this model describes a rotating rotor but not the Helium atom, and that it must be abandoned. 


\section{Acknowledgements}

I thank Dr Andreas Rüetschi-Isler for his critical objections enabling a distinct assessment and David Kummer for performing the 3D-animation and their freeze images.

\section{Conflicts of Interest}

The author declares no conflicts of interest regarding the publication of this paper.

\section{References}

[1] Allmendinger, T. (2018) The Spherical Atom Model of Helium Based on the Theorem of Niels Bohr. Journal of Applied Mathematics and Physics, 7, 172-180.

https://file.scirp.org/Html/15-1721414_90090.htm https://doi.org/10.4236/jamp.2019.71015

[2] Kimball, G.E. (1940) Directed Valence. Journal of Chemical Physics, 8, 188. https://doi.org/10.1063/1.1750628

[3] Bohr, N. (1913) On the Constitution of Atoms and Molecules. The London, Edinburgh, and Dublin Philosophical Magazine and Journal of Science, 26, 1-25. https://doi.org/10.1080/14786441308634955

[4] Uhlenbeck, G.E. and Goudsmith, S. (1925) Ersetzung der Hypothese vom unmechanischen Zwang durch eine Forderung bezüglich des inneren Verhaltens jedes einzelnen Elektrons. Naturwissenschaften, 13, 953-954.

https://doi.org/10.1007/BF01558878

[5] Uhlenbeck, G.E. and Goudsmith, S. (1926) Spinning Electrons and the Structure of Spectra. Nature, 117, 264-265. https://doi.org/10.1038/117264a0

[6] Allmendinger, T. (2018) The Elucidation of the Ground State in the H-Atom-Model of Niels Bohr and Its Application on the Bond-Length Computation in the $\mathrm{H}_{2}$-Molecule. International Journal of Molecular and Theoretical Physics, 2, 1-10. https://symbiosisonlinepublishing.com/molecular-theoretical-physics/molecular-th eoretical-physics09.pdf https://doi.org/10.15226/2576-4934/2/1/00109

[7] Allmendinger, T. (2018) The Atom Model of Helium and of Neon Based on the Theorem of Niels Bohr. Journal of Applied Mathematics and Physics, 6, 1290-1300. https://www.scirp.org/Journal/PaperInformation.aspx?PaperID=85615 https://doi.org/10.4236/jamp.2018.66108 